\title{
Peptic duodenitis-does it exist in the second part of the duodenum?
}

\author{
N Leonard, C F Feighery, D O’B Hourihane
}

\begin{abstract}
Aim-To re-evaluate all patients diagnosed histologically as having peptic duodenitis who had known endomysial antibody (EMA) test results to find out whether they would still be classified as peptic duodenitis on histological analysis and to review their subsequent clinical course.

Methods-All mucosal biopsy specimens of the second part of the duodenum which were reported as showing features of peptic duodenitis and on which a serum EMA test had been done between January 1990 and January 1995 were reviewed. The number of intraepithelial lymphocytes (IELs) per 500 epithelial cells was also counted. The cases were re-assigned to one of three clinical categories: normal, coeliac disease or peptic duodenitis. Clinical details were reviewed for any cases where the re-assigned diagnosis and the EMA test result did not correlate.
\end{abstract}

Results-Of the 24 cases, 21 showed a correlation between morphology and immunology - that is, if the biopsy specimen was characteristic of coeliac disease, the EMA was positive and if the biopsy specimen was normal or characteristic of peptic duodenitis, the EMA was negative. Three cases had a negative correlation: two had a positive EMA test but a biopsy diagnosis of peptic duodenitis and one had a normal duodenal biopsy specimen with a positive EMA test. On review of their clinical details, two of the three patients were diagnosed with coeliac disease and the other with silent coeliac disease. EMA test results and IEL counts correlated with the final diagnosis in all cases.

Conclusions-The diagnosis of peptic duodenitis on biopsy specimens of the second part of the duodenum was not substantiated in $92 \%$ of cases. On review of 24 cases, a histological diagnosis of peptic duodenitis was reached in four. In difficult cases, the histological appearances should be correlated with the EMA test result and the IEL count. Correlation of this kind should leave no cases of coeliac disease undiagnosed.

(F Clin Pathol 1997;50:54-58)

Keywords: coeliac disease; peptic duodenitis; endomysial antibody.

Florid cases of coeliac disease with a flat mucosa, notably raised intraepithelial lym- phocytes (IELs) and a positive endomysial antibody (EMA) test are easy to diagnose on biopsy specimens from the second part of the duodenum. Although jejunal biopsy specimens would be easier to interpret, several studies have shown that samples from the second part of the duodenum are satisfactory as well as being useful in excluding other pathology. ${ }^{1}$ Cases of peptic duodenitis which have ulcer formation, polymorphonuclear abscesses and abundant gastric metaplasia are also relatively easy to diagnose. However, in our service, there is a group of patients who have had a histological diagnosis of peptic duodenitis but who have subsequently developed equivocal clinical or immunological findings suggesting that they might have coeliac disease rather than peptic duodenitis. The aim of this study was to re-evaluate all patients histologically diagnosed as peptic duodenitis who also had known EMA test results to establish whether they would still be classified as peptic duodenitis on histological analysis. The subsequent clinical course of these patients was also reviewed.

\section{Methods}

Between January 1990 and January 1995, 6200 duodenal biopsy specimens were taken, 196 of which were indicative of coeliac disease. As coeliac disease is common in Ireland, a serum EMA test is often done on patients with gastrointestinal symptoms when coeliac disease is not necessarily the first clinical differential. All mucosal biopsy specimens from the second part of the duodenum which were reported as showing features of peptic duodenitis and which had an accompanying serum EMA test done between January 1990 and January 1995 were reviewed. Twenty four biopsy specimens fulfilled these criteria. Most of these specimens would have been reviewed at weekly clinicopathological meetings so that a diagnosis of either coeliac disease or peptic duodenitis would not be made without some clinical correlation. An EMA test was requested at these meetings if not already available.

Sections, $4 \mu \mathrm{m}$ thick, were stained with haematoxylin and eosin, periodic acid Schiff (PAS) and Warthin-Starry silver stains. Sections were reviewed with regard to the following features: villous atrophy; crypt hyperplasia; gastric metaplasia (as assessed by staining with PAS); chronic inflammation; acute inflammation in lamina propria, crypts and surface epithelium; and presence or absence of Helicobacter-like organisms (assessed using Warthin-Starry stain). Villous atrophy was regarded as present of Histopathology,

Northwick Park/St Mark's

NHS Trust,

Accepted for publication 22 October 1996 
Table 1 Clinical details

\begin{tabular}{|c|c|c|c|c|c|c|c|c|c|}
\hline $\begin{array}{l}\text { Case } \\
\text { number }\end{array}$ & $\begin{array}{l}\text { Age } \\
\text { (years) }\end{array}$ & Sex & Symptoms & $\begin{array}{l}\text { Appearance of } \\
\text { villi }\end{array}$ & $\begin{array}{l}\text { Polymorph } \\
\text { infiltrate }\end{array}$ & $\begin{array}{l}\text { EMA test } \\
\text { result }\end{array}$ & IEL count & $\begin{array}{l}\text { Histological } \\
\text { diagnosis }\end{array}$ & Final diagnosis \\
\hline 1 & 20 & $M$ & Diarrhoea & Nearly flat & + & Positive & $20-40$ * & Peptic duodenitis & Coeliac disease \\
\hline 2 & 30 & $\mathrm{~F}$ & Diarrhoea & flat & ++ & Positive & 28 & Peptic duodenitis & Coeliac disease \\
\hline 3 & 73 & $\mathrm{~F}$ & Anaemia, dyspepsia & Normal & + & Positive & 30 & Normal & Coeliac disease \\
\hline 4 & 22 & $\mathrm{~F}$ & Dyspepsia & Normal & ++ & Negative & 5 & Peptic duodenitis & Peptic duodenitis \\
\hline 5 & 50 & $\mathrm{~F}$ & Dyspepsia & Some stubby & - & Negative & 8 & Peptic duodenitis & Peptic duodenitis \\
\hline 6 & 70 & $\mathrm{M}$ & Anaemia, dyspepsia & Normal & - & Negative & 13 & Normal & Normal \\
\hline 7 & 24 & $\mathrm{~F}$ & Weight loss, abdominal pain & Normal & - & Negative & 12 & Normal & Normal \\
\hline 8 & 20 & $\mathrm{~F}$ & Anaemia & Normal & - & Negative & 5 & Normal & Normal \\
\hline 9 & 73 & $\mathrm{~F}$ & Dyspepsia & Normal & - & Negative & 12 & Normal & Normal \\
\hline 10 & 20 & $\mathrm{M}$ & Mouth ulcers & Normal & - & Negative & 15 & Normal & Normal \\
\hline 11 & 57 & $\mathrm{~F}$ & Dyspepsia & Normal & - & Negative & 5 & Normal & Normal \\
\hline 12 & & $\mathrm{M}$ & Dyspepsia & Flat & - & Positive & 60 & Coeliac disease & Coeliac disease \\
\hline 13 & 63 & $M$ & Anaemia & Flat & + & Positive & 32 & Coeliac disease & Coeliac disease \\
\hline 14 & 44 & $\mathrm{~F}$ & Dyspepsia & Flat & + & Positive & 60 & Coeliac disease & Coeliac disease \\
\hline 15 & 35 & $\mathrm{~F}$ & Dyspepsia & Flat & - & Positive & * & Coeliac disease & Coeliac disease \\
\hline 16 & 39 & $\mathrm{~F}$ & Anaemia & Flat & + & Positive & 18 & Coeliac disease & Coeliac disease \\
\hline 17 & 57 & $M$ & Dyspepsia & Stubby & - & Positive & 20 & Coeliac disease & Coeliac disease \\
\hline 18 & 52 & $\mathrm{~F}$ & Dyspepsia & Flat & - & Positive & 40 & Coeliac disease & Coeliac disease \\
\hline 19 & 45 & $\mathrm{~F}$ & Anaemia & Flat & - & Positive & 50 & Coeliac disease & Coeliac disease \\
\hline 20 & 54 & $\mathrm{~F}$ & Anaemia & Abnormal & - & Positive & 36 & Coeliac disease & Coeliac disease \\
\hline 21 & 20 & $M$ & ? & Flat & + & Positive & 100 & Coeliac disease & Coeliac disease \\
\hline 22 & 25 & $\mathrm{~F}$ & Diarrhoea, anaemia & Stubby & + & Positive & 19 & Coeliac disease & Coeliac disease \\
\hline 23 & 40 & $M$ & Dyspepsia, diarrhoea & Normal & + & Positive & 50 & Coeliac disease & Coeliac disease \\
\hline 24 & 48 & $M$ & Anaemia & Stubby & - & Positive & 60 & Coeliac disease & Coeliac disease \\
\hline
\end{tabular}

$\star$ Difficult to interpret.

if the villus to crypt ratio was less than $1: 1$. This is because the normal ratio in the duodenum is less than in the jejunum. In specimens without muscularis mucosae a ratio as low as $1: 1$ is accepted as normal ${ }^{12}$ although the normal ratio tends to be between 3:1 and 5:1. The number of IELs per 500 epithelial cells was also counted.

The histopathological features used to diagnose coeliac disease include partial to subtotal villous atrophy with crypt hyperplasia and surface epithelial damage. The lamina propria may contain increased numbers of white cells, mainly plasma cells. ${ }^{3}$

Criteria for the diagnosis of peptic duodenitis in the first part of the duodenum have been established. ${ }^{4}$ They include irregularity and shortening of villi with oedema in the lamina propria. Damage to the superficial epithelium involves flattening and basophilia, absence of the brush border, gastric metaplasia, invasion of polymorphs, and erosions. An increased number of lymphocytes, plasma cells and polymorphs may be present in the lamina propria. Peptic duodenitis can be further classified into three grades. Grade 1 or mild duodenitis is associated with essentially normal architecture and surface epithelium. There is an increased number of lymphocytes and plasma cells in the lamina propria. In grade 2 or moderate duodenitis, abnormal surface epithelium with occasionally deformed and shortened villi with focal gastric metaplasia is found. The features of grade 3 or severe duodenitis include erosion of surface epithelium with florid inflammation, deformed villi and extensive gastric metaplasia. It has been suggested recently that grade 1 duodenitis should be classified within normal limits. ${ }^{1}$ However, this grade has been retained in our study as the specimens were originally reported when this classification was in use.

Slides were examined and re-assigned as normal, peptic duodenitis or coeliac disease without knowledge of clinical course or EMA test results. Following this, EMA test results were collected and compared with the histo- logical diagnosis and the IEL count. Some of the earlier cases had $\alpha$-gliadin antibody (AGA) test results. Clinical data were collected on all cases.

\section{Results}

On review of the clinical details (table 1) it was apparent that this group had been difficult to diagnose clinically, often having multiple biopsies done over a period of several years and numerous discussions at clinicopathological meetings.

After morphological analysis the cases were re-assigned to the following diagnostic categories: 13 as coeliac disease; four as peptic duodenitis; and seven as normal.

Of the 13 cases of coeliac disease, nine had flat mucosa and four had stubby villi. Four patients had gastric metaplasia in $10 \%$ or less of the surface epithelium. All had chronic inflammatory cells in the lamina propria of mild to moderate extent. Eight had prominent polymorphs present in this infiltrate, five of whom had polymorph infiltration of surface epithelium.

The seven patients re-assigned as normal had normal architecture with mild chronic inflammation and could have been classified as grade 1 peptic duodenitis. The only clear abnormality was the presence of polymorphs in one patient who had been treated for coeliac disease in the past (case 3; see later).

Of the four cases of peptic duodenitis, two had a flat mucosa (cases 1 and 2) and two had normal villi. Gastric metaplasia involving between 5 and $20 \%$ of the surface epithelium was found in two patients (cases 1 and 2). All showed chronic inflammation of the lamina propria of moderate extent and all contained polymorphs both in the lamina propria and infiltrating the surface epithelium. No erosions were found in any of the patients. All were classified as grade 2 peptic duodenitis.

Of the 24 cases, the EMA test was positive in 16 and negative in eight. All 13 cases of coeliac disease were EMA positive. One of the seven 
re-assigned normal cases was EMA positive (case 3). Two of the four histologically re-assigned cases of peptic duodenitis were EMA positive (cases 1 and 2). When IEL counts were evaluated they were uniformly high in the patients with coeliac disease, with a mean (SD) of 50\% (251 (111) per 500 enterocytes). The normal subjects had a mean (SD) of $14 \%(71(41))$ as did the cases of peptic duodenitis (71 (50)). However, there was a scattered distribution of IEL counts in those with peptic duodenitis. The two cases with positive EMA test results had IEL counts of $28 \%$ and $40 \%$, whereas the two EMA negative cases had IEL counts of $5 \%$ and $8 \%$.

None of the patients were infected with Helicobacter, as assessed on Warthin-Starry stained sections. Serology for Helicobacter was not available when most of the biopsy specimens were taken.

A correlation was found between morphology and immunology in 21 of the 24 patientsthat is, if the biopsy specimen was characteristic of coeliac disease, the EMA test was positive and if the biopsy specimen was normal or characteristic of peptic duodenitis, the EMA test was negative. A negative correlation was found in three cases and their clinical details are given later: two were EMA positive but had a biopsy diagnosis of peptic duodenitis and one had a normal duodenal biopsy specimen with a positive EMA result.

\section{Case reports}

CASE 1

Re-assigned histological diagnosis-peptic duodenitis: normal and stubby villi with $20 \%$ gastric metaplasia, prominent polymorphs in the lamina propria and epithelium. IEL count: 20-40\% (difficult technically).

A 19 year old man presented with a four month history of diarrhoea. The duodenal biopsy specimen showed peptic duodenitis and his EMA test was positive. The patient was started on a high roughage diet and his diarrhoea resolved. However, nine months later the EMA was still positive, intermittent diarrhoea was still present and he had noticed a failure to gain weight. The final diagnosis was probable coeliac disease.

\section{CASE 2}

Re-assigned histological diagnosis-peptic duodenitis: flat mucosa with $5 \%$ gastric metaplasia, prominent polymorphs in the lamina propria, surface epithelium and infiltrating into crypts (fig 1). IEL count: $28 \%$.

A 30 year old woman presented with abdominal pain. She had a childhood history of coeliac disease and had stopped her gluten-free diet at the age of 12 . She was restarted on a gluten-free diet with relief of her symptoms. A repeat biopsy specimen showed continuing duodenal inflammation. The EMA test was positive. The final diagnosis was probable coeliac disease.

CASE 3

Re-assigned histological diagnosis-normal: mainly normal architecture with some stubby

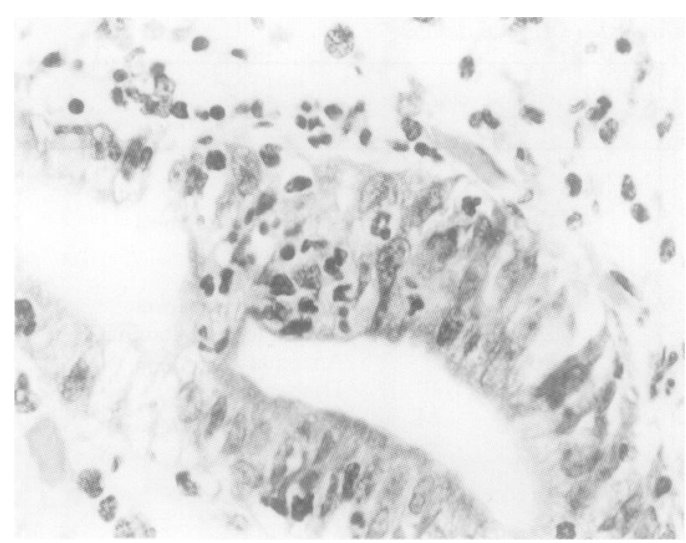

Figure 1 Extensive polymorph infiltration of a crypt in a patient with a re-assigned diagnosis of peptic duodenitis but with a positive EMA result and clinical history suggestive of coeliac disease (case 2).

villi, minimal chronic inflammation and polymorphs in the lamina propria. There was no gastric metaplasia. IEL count: $30 \%$.

A 73 year old woman presented with iron deficiency anaemia. She had reflux oesophagatis, raised AGA and subtotal villous atrophy on biopsy. She was started on a gluten-free diet. Her haemoglobin concentrations increased. Six months later, a further biopsy specimen (reviewed here) was equivocal/normal. However, AGA were still raised and the EMA test was positive. The final diagnosis was treated coeliac disease.

In view of the above clinical histories and EMA test results it seems much more likely that cases 1, 2 and 3 have coeliac disease.

Two of the original 24 cases of peptic duodenitis retained their original diagnosis. The clinical features of these cases are reviewed below.

CASE 4

Re-assigned histological diagnosis-peptic duodenitis: normal villi with $20 \%$ gastric metaplasia, moderate chronic inflammation in the lamina propria, with polymorphs present in the lamina propria and surface epithelium. IEL count: $5 \%$

A 22 year old man presented with a six year history of intermittent dyspepsia, heartburn and vomiting. At gastroscopy, he had a large ulcer in the anterior wall of the first part of the duodenum, which contained Helicobacter-like organisms. AGA were notably raised (32 units/ $\mathrm{ml})$. The patient was treated with omeprazole and four months later his ulcer had healed and he had no further symptoms. A biopsy specimen of the second part of the duodenum was normal at this time, the EMA test was negative but AGA were still raised. The final diagnosis was peptic duodenitis.

\section{CASE 5}

Re-assigned histological diagnosis-peptic duodenitis: normal villi with no gastric metaplasia. There was moderate chronic inflammation with prominent polymorphs in the lamina propria, crypts and surface epithelium. IEL count: $8 \%$.

A 50 year old woman presented with a five year history of epigastric pain, nausea, belching, and anxiety. The duodenal biopsy speci- 
men showed features of peptic duodenitis and the EMA test was negative. The final diagnosis was peptic duodenitis.

\section{Discussion}

These results indicate that, in our service, a diagnosis of peptic duodenitis is not reliable. Twenty two (92\%) of 24 initial diagnoses of peptic duodenitis were reclassified as coeliac disease or normal following more detailed evaluation. All of the biopsy specimens had been taken from the second part of the duodenum and it is noteworthy that the criteria used to diagnose peptic duodenitis originate from descriptions of specimens from the first part of the duodenum. ${ }^{5}$ It was not mentioned whether the features are similar in the second part of the duodenum or indeed whether peptic duodenitis exists in this region of the duodenum. The present study also confirms recent suggestions that grade 1 duodenitis should really be classified as within normal limits. ${ }^{4}$

Bayless et al noted that peptic duodenitis may be confused with coeliac disease as these entities share many histological features, including abnormal architecture, damage to surface epithelium and a prominent inflammatory process. It has been suggested that if the diseases coexist, the presence of peptic duodenitis would prevent a complete response to a gluten-free diet. Differentiation between the two conditions could be achieved by examining and comparing biopsy specimens of both the first and second parts of the duodenum. Jeffers and Hourihane ${ }^{7}$ found that polymorph infiltration and gastric metaplasia could occur in coeliac disease and should not be used to differentiate between coeliac disease and peptic duodenitis.

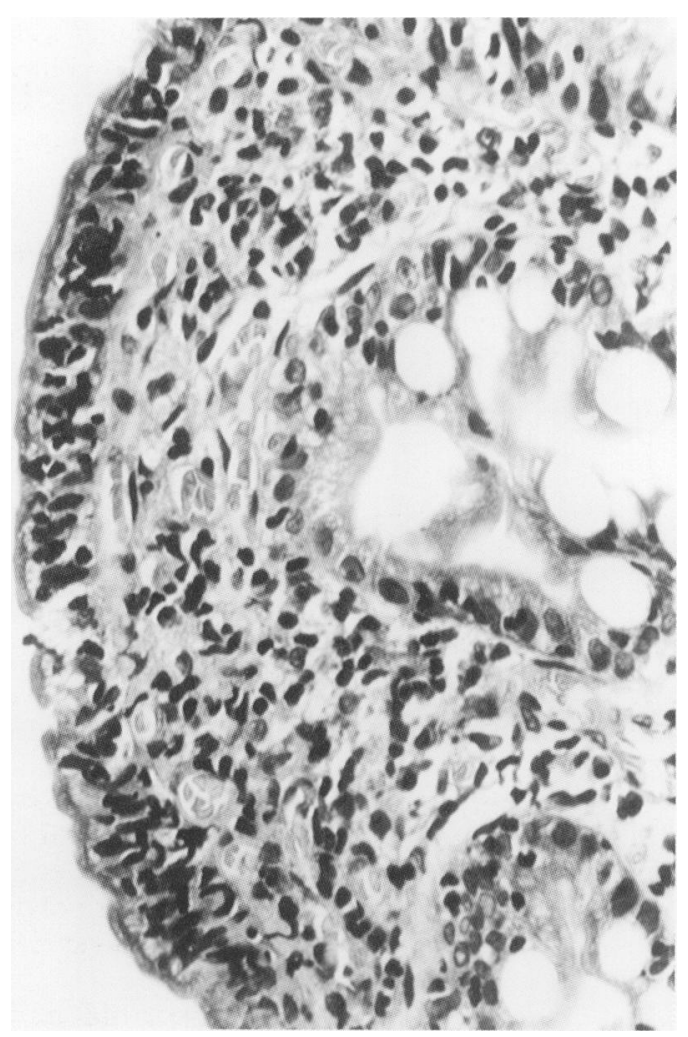

Figure 2 A case of coeliac disease showing surface epithelium unsuitable for IEL counting.
Hence, the problem of differentiating between peptic duodenitis and coeliac disease is not a new one. In the present study the diagnosis of peptic duodenitis from biopsy specimens of the second part of the duodenum was substantiated in only two of the 24 cases. Therefore, caution should be exercised before making this diagnosis on tissue from this section of the duodenum.

It was emphasised previously that the IEL count may be a crucial discriminating factor in cases with an uncertain diagnosis of coeliac disease. This was certainly the case in our series where an IEL count of greater than $18 \%$ was present in all cases found to have coeliac disease. IEL counts always correlated with the EMA test result and in difficult cases were the discriminating feature. Raised IEL counts may be the earliest abnormality seen in coeliac disease, which has been described as 'high density intraepithelial lymphocyte enteropathy'. ${ }^{8}$ It is important to count objectively the number of IELs/300-500 epithelial cells as their number may vary greatly between one villus and the next.

On occasion, IEL counts may be difficult technically, as can be seen from fig 2 . If the section is poor it is impossible to do an accurate count. In many cases, there may be very little surface epithelium present on what is otherwise an adequate specimen (fig 3 ). If there is extensive gastric metaplasia there may be very little small intestinal epithelium present on which to do a count (fig 4). One surprising feature in our study was that in several cases IELs had infiltrated the gastric metaplastic epithelium (fig 4). One solution to these technical difficulties may be to use Bouin's solution as a fixative as it produces better preservation of nuclear detail than formalin.

Several biopsy specimens were re-classified as normal and this highlights the problem of interpreting how much lamina propria inflammation can be present in a normal specimen. Gastric metaplasia has also been reported in a

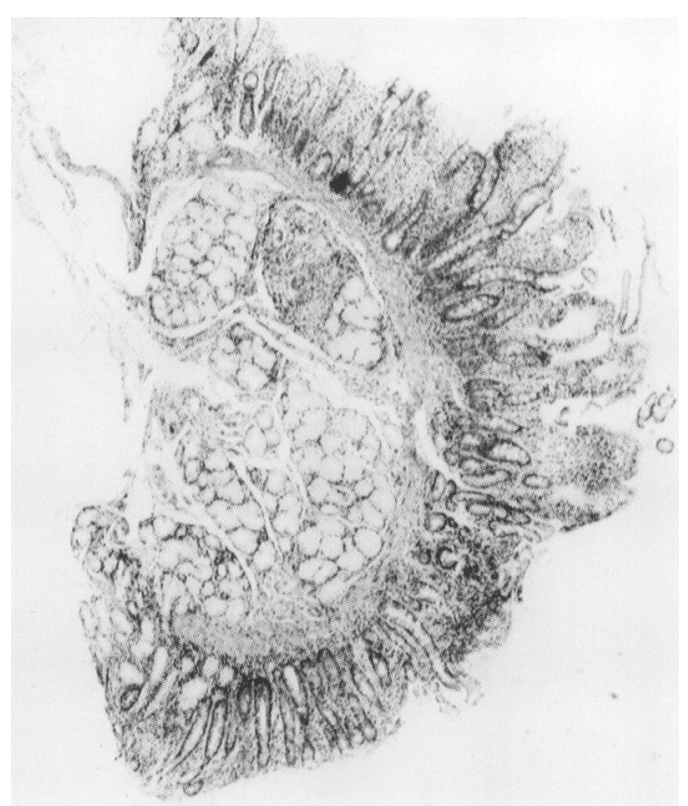

Figure 3 Biopsy specimen with very little surface epithelium. 


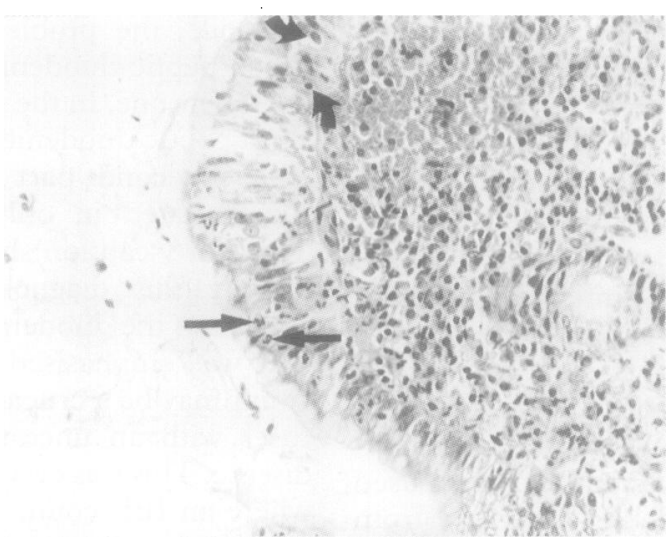

Figure 4 Extensive gastric metaplasia which takes up most of the surface epithelium. The straight arrows indicate polymorphs and the curved arrows lymphocytes present in gastric-type epithelium.

large percentage of specimens from the first part of the duodenum from normal volunteers, ${ }^{9}$ although there have been no studies of the second part of the duodenum. The differential diagnosis of coeliac disease and peptic duodenitis is not the only differential involved, as Crohn's disease, food intolerances and other non-specific systemic disorders can affect this part of the duodenum.

In summary, diagnosis of peptic duodenitis on biopsy specimens of the second part of the duodenum was not substantiated in $92 \%$ of cases. On histological review of 24 cases only four were diagnosed histologically as peptic duodenitis. Moreover, when EMA test results and clinical details were assessed, the diagnosis of peptic duodenitis could be substantiated in only two of these four patients. In the remain- ing two patients, a diagnosis of coeliac disease was reached. To some extent, the histological diagnosis of peptic duodenitis was used as an alternative diagnosis to coeliac disease in our service.

In all of our cases the EMA test result correlated with the final combined diagnosis. This confirms previous reports of the specificity of EMA for coeliac disease. ${ }^{10}$ IEL counts are also extremely useful in the diagnosis of coeliac disease and should be counted in all patients. In difficult cases, the histological appearances should be correlated with the EMA test result and the IEL count. Correlation of this kind should leave no cases of coeliac disease undiagnosed.

1 Day DW. Biopsy pathology of the oesophagus, stomach and duodenum. London: Chapman and Hall, 1995.

2 Segal GH, Petras RE. In: Sternberg SS, ed. Histology for pathologists. New York: Raven Press, 1992

3 Lee FD. Malabsorption. In: McGee JO'D, Isaacson I, Wright NA, eds. Textbook of pathology. Oxford: Oxford University Press, 1992.

4 Sipponen P. Chapter 25; part IV. In: Whitehead R, ed. Gastrointestinal and oesophageal pathology. 2nd edn. Edinburgh Churchill Livingstone, 1995.

5 Trier JS. Morphology of the gastric mucosa in patients with ulcer diseases. Am $\mathcal{f}$ Dig Dis $1976 ; 21: 138-40$.

6 Bayless TM, Kapelowitz RF, Shelley WM, Ballinger WF Bayless TM, Kapelowitz RF, Shelley WM, Ballinger WF,
Hendrix TR. Intestinal ulceration: a complication of Hendrix TR. Intestinal ulceration: a complication
coeliac disease. $N$ Engl $₹$ Med 1967;276:996-1002.

7 Jeffers MD, Hourihane DO'B. Coeliac disease with histological features of peptic duodenitis: Value of assessment of intraepithelial lymphocytes. F Clin Pathol 1993;46: 420-4.

8 Ferguson A, Arranz E, O'Mahony S. Clinical and pathological spectrum of coeliac disease-active, silent, latent, potential. Gut 1993;34:150-1.

9 Kreunig J, Bosman FT, Kuiper G, van der Val AM, Lindeman J. Gastritis and duodenal mucosa in 'healthy' individuals: an endoscopic and histopathological study of individuals: an endoscopic and histopatholo

50 volunteers. $\mathcal{F}$ Clin Pathol 1978;31:69-77.
10 Hallstrom O. Comparison of IgA-class reticulin and endomysium antibodies in coeliac disease and dermatitis herpetiformis. Gut 1989;30:1225-32. 